\title{
Reporte de Caso: Origen del Músculo Piriforme a Través de Dos Cabezas en Forma de Bíceps, Propicia División del Nervio Isquiático en Nervio Fibular Común y Nervio Tibial
}

\author{
Case Report: Origin of the Piriformis Muscle Through Two Heads in the Form of Biceps, \\ Favorable Division of the Sciatic Nerve in the Common Fibular Nerve and Tibial Nerve
}

\author{
Gonzalo Tiznado-Matzner'; Sandra Bucarey-Arriagada ${ }^{1} \&$ Rodrigo Lizama Pérez ${ }^{2}$
}

TIZNADO-MATZNER, G.; BUCAREY-ARRIAGADA, S. \& LIZAMA, R. P. Reporte de Caso: Origen del músculo piriforme a través de dos cabezas en forma de bíceps, propicia división del nervio isquiático en nervio fibular común y nervio tibial. Int. J. Morphol., 38(1):199-202, 2020.

RESUMEN: El músculo piriforme es un músculo pelvitrocantérico que recibe su nombre debido a su forma de pera, cuyo origen es de varios fascículos que se encuentran entre los forámenes anteriores del sacro, correspondiente a las segunda, tercera y cuarta vértebra. Estos fascículos se funden constituyendo un músculo aplanado, que se inserta en el trocánter mayor del fémur. Presenta una relación bien conocida con el nervio isquiático, el cual comúnmente emerge hacia la región glútea por el margen inferior de este músculo, sin embargo a través del tiempo, autores han descrito variaciones del paso de este nervio que podrían asociarse a alguna patología de compresión del nervio isquiático. En una disección rutinaria de dos individuos formolizados, uno femenino y otro masculino de la región glútea, encontramos que el músculo piriforme se originaba a través de dos cabezas, cada una con su propia fascia que se unían en un vientre común, en forma de bíceps y a través de un tendón cilíndrico se insertaban en la parte medial del trocánter mayor del fémur. El nervio isquiático se encontraba dividido, el nervio fibular común emergía a la región glútea a través de las cabezas, en tanto el nervio tibial por el margen inferior del músculo piriforme. Es importante comunicar las variaciones anatómicas para complementar el conocimiento de las mismas, las que pueden explicar ciertos trastornos físicos y dolorosos como el denominado síndrome del músculo piriforme.

PALABRAS CLAVE: Músculo piriforme; Nervio isquiático; Variaciones.

\section{INTRODUCCIÓN}

El músculo piriforme deriva su nombre por su forma de pera, originándose en la cara anterior del sacro alrededor de los forámenes sacros formado por tres o cuatro fascículos, más o menos diferenciados a un nivel correspondiente a las vértebras sacras segunda, tercera y cuarta (Testut \& Latarjet, 1972). Se funden constituyendo un sólo músculo aplanado, que abandona la pelvis a través del foramen isquiático mayor, para conformar un tendón cilíndrico que se inserta en la parte media del margen superior del trocánter mayor fémur (Moore \& Dalley, 2007). Presentan una estrecha relación con el nervio isquiático que emerge a la región glútea por el margen inferior del músculo piriforme, el cual puede presentar variaciones en su paso. Autores como Vicente et al. (2007), Boyajian-O`Neill et al. (2008), Ilizaliturri et al. (2018), Ro \& Edmonds (2018), relacionan la variación del paso del nervio isquiático a través del músculo piriforme a la patología denominada síndrome del músculo piriforme, en el cual este nervio podría verse comprimido por hipertrofia de este músculo.

En el presente artículo se describe una variación anatómica que corresponde a la conformación del músculo piriforme en la pelvis y a la emergencia a la región glútea del nervio isquiático que compromete al músculo piriforme.

\section{REPORTE DE CASO}

La variación anatómica fue observada durante la disección rutinaria de la región glútea de dos cadáveres formolizados, uno en la pelvis izquierda de una muestra de sexo femenino (Fig. 1) y el otro en la pelvis derecha de una muestra de sexo masculino (Fig. 2), pertenecientes al labo-

\footnotetext{
${ }^{1}$ Instituto de Anatomía, Histología y Patología, Facultad de Medicina. Universidad Austral de Chile, Valdivia, Chile.

${ }^{2}$ Departamento de Ciencias Morfológicas, Facultad de Medicina y Ciencia, Universidad San Sebastián, General Lagos 1163, Valdivia 5090000, Chile.
} 
ratorio de Anatomía Humana de la Universidad Austral de Chile. En la disección, tras retirar la piel y tejido adiposo, se separó el músculo glúteo máximo, pudiéndose observar claramente los músculos pelvitrocantéricos profundos.

En ambos casos se apreció que el músculo piriforme se originaba en la cara anterior del sacro a través de dos cabezas. La cabeza superior se originaba en la cara anterior de las dos primeras vértebras sacras, en tanto la cabeza inferior lo hacía desde la tercera y cuarta vértebra sacra. Ambas cabezas se unían lateralmente formando el vientre muscular

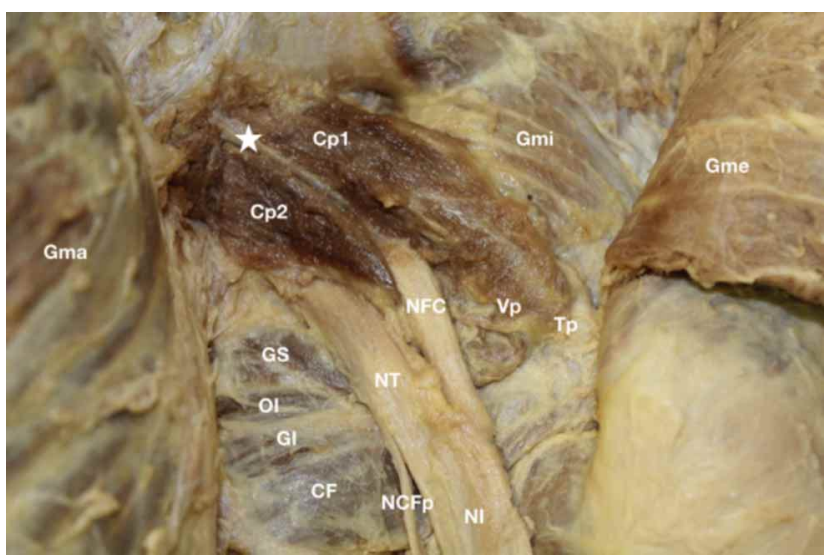

Fig. 1. Músculo piriforme de pelvis de mujer que es originado por dos cabezas; con emergencia del nervio fibular común entre ellas. Cabeza superior del músculo piriforme (Cp1); Cabeza inferior del músculo piriforme (Cp2); fascia de las cabezas del músculo piriforme (Estrella blanca); Vientre del músculo piriforme (Vp); Tendón del músculo piriforme (Tp); Nervio fibular común (NFC); Nervio Tibial (NT); Nervio isquiático (NI); Nervio cutáneo femoral posterior (NCFp), Músculo glúteo máximo (Gma); Músculo glúteo medio (Gme); Músculo glúteo mínimo (Gmi); Músculo gemelo superior (GS); Músculo obturador interno (OI); Músculo gemelo inferior (GI); Músculo cuadrado femoral (CF).

\section{DISCUSIÓN}

A través del tiempo las variaciones anatómicas, especialmente de estructuras vasculonerviosas y musculares aparecen regularmente durante las disecciones de rutina. Esto se puede evidenciar en la literatura donde varios autores han plasmado en textos lo encontrado a través del tiempo.

Debido a su ubicación, el músculo piriforme se utiliza como elemento de referencia de la región glútea, y proporciona la clave para entender las relaciones de esta región, porque determina los nombres de los nervios y los vasos sanguíneos, como por ejemplo, el nervio y los vasos glúteos superiores emergen superiores al músculo, por su lado el nervio y los vasos glúteos inferiores emergen inferiores al músculo (Moore \& Dalley). Claramente igual sirve para y finalizando en un solo tendón que se insertaba en la parte media del margen superior del trocánter mayor del fémur. Cabe resaltar, que cada cabeza se encontraba cubierta por su propia fascia, que finalmente se fundían al formarse el vientre muscular y lateralmente el tendón común, a modo de músculo bíceps. Entre ambas cabezas se observó que emergía el nervio fibular común, en tanto que el nervio tibial aparece por el margen inferior del músculo, para finalmente unirse y conformar el nervio isquiático. Una vez emergido el nervio fibular común, es donde las cabezas del piriforme se unen formando el vientre muscular.

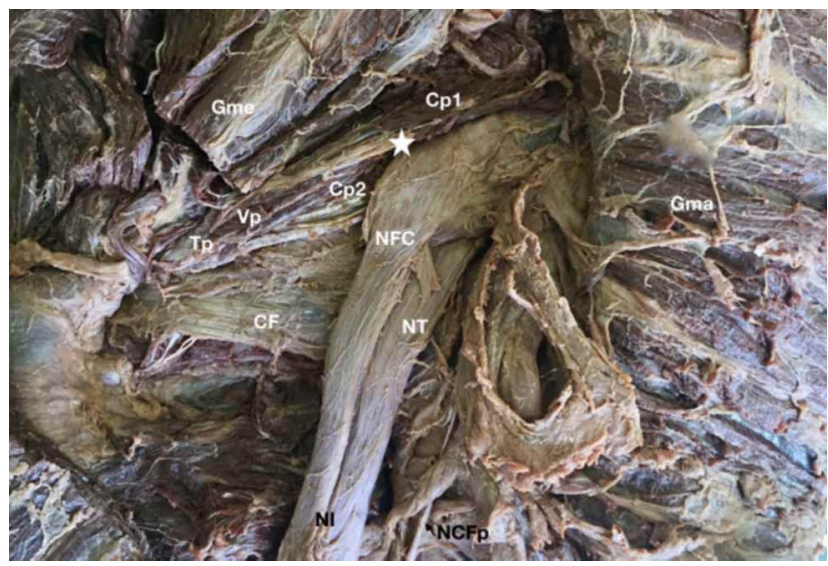

Fig. 2. Músculo piriforme de pelvis de hombre que es originado por dos cabezas; con emergencia del nervio fibular común entre ellas. Cabeza superior del piriforme (Cp1); Cabeza inferior del piriforme (Cp2); Fascia de las cabezas del músculo piriforme (Estrella blanca); Vientre del músculo piriforme (Vp); Tendón del músculo piriforme (Tp); Nervio fibular común (NFC); Nervio tibial (NT); Nervio isquiático (NI); Nervio cutáneo femoral posterior (NCFp); Músculo glúteo máximo (Gma); Músculo glúteo medio (Gme); Músculo cuadrado femoral (CF).

referenciar la emergencia hacia posterior del nervio isquiático, nervio del cual se han reportado varias formas de emerger hacia la región glútea, lo que ha dado un gran número de publicaciones que incluso lo vinculan a la patología conocida como el síndrome del músculo piriforme, en el cual se produce una compresión del nervio isquiático, por presunta hipertrofia del músculo piriforme.

Beaton \& Anson (1937) clasificaron en 6 tipos la relación entre el músculo piriforme y el nervio isquiático (Tabla I).

Nuestros casos corresponden al tipo II. Sin embargo, nosotros no coincidimos con Beaton \& Anson y Jacomo et 
al. (2014) quienes indicaron que el músculo piriforme genera un músculo accesorio, sino que es el mismo músculo que se origina a través de dos cabezas independientes, como indicamos anteriormente, cada una con su propia fascia, que provienen de las vértebras primera, segunda, tercera y cuarta del sacro, que es el origen típico de este músculo. El nervio fibular común, al emerger entre las cabezas, no pasa entre las fibras de este músculo, por lo cual puede acceder por esta zona sin verse afectado por la hipertrofia de las fibras musculares.

Natsis et al. (2014), haciendo una revisión de 25 autores, utilizando la clasificación de Beaton \& Anson, encontraron, claramente, que el tipo I era la más común, en tanto, el tipo Tipo V y VI los más raros (Tabla II).

Las diferentes variaciones del paso del nervio isquiático por el músculo piriforme podría causar la compresión de algún componente de este nervio, llamado síndrome del músculo piriforme, como refiere Santos et al. (2009) y Ro \& Edmonds, quienes aducen que tiene una incidencia de alrededor del 6-8 \% en pacientes con lumbalgia, entre cuyos síntomas además del dolor de espalda, presentarían dolor a la sedestación, bipedestación y/o decúbito supino por más de 15 minutos y parestesias en la parte posterior del miembro inferior. No obstante, Kirschner et al. (2009) han señalado que no está claro que estas variaciones anatómicas sean las responsables o contribuyan a esta patología, debido a que algunos pacientes asintomáticos presentan estas variaciones y algunos sintomáticos no las presentan. Muñoz (2004), Polesello et al. (2013) y Kraus et al. (2016) a través de resonancia nuclear magnética evidenciaron casos de pacientes que sufrían del síndrome del músculo piriforme, en el mismo lugar donde se observaba hipertrofia del músculo piriforme.

Considerando lo anterior, es posible concluir que el nervio fibular común al emerger por entre las cabezas del músculo piriforme y no pasando entre sus fibras, no tendría grandes posibilidades de ser comprimido por el músculo, lo que ocurriría probablemente en paciente que muestran esta variación, pero son asintomáticos generando una incidencia tan baja en el llamado síndrome del piriforme. Sin embargo es más probable que se produzca un compresión de este nervio si es que pasa directamente entre las fibras musculares.
Tabla II. Incidencia de los tipos de variaciones de la relación entre el músculo piriforme y nervio isquiático realizado utilizando la clasificación de Beaton \& Anson (1937) por Natsis et al. (2014).

\begin{tabular}{lr}
\hline Tipos & Incidencia \\
\hline Tipo I & $82,5 \%$ \\
Tipo II & $11,7 \%$ \\
Tipo III & $2 \%$ \\
Tipo IV & $0,68 \%$ \\
Tipo V & $0,12 \%$ \\
Tipo VI & $0,13 \%$ \\
\hline
\end{tabular}

TIZNADO-MATZNER, G.; BUCAREY-ARRIAGADA, S. \& LIZAMA, R. P. Case Report: Origin of the piriformis muscle through two heads in the form of biceps, favorable division of the sciatic nerve in the common fibular nerve and tibial nerve. Int. J. Morphol., 38(1):199-202, 2020.

SUMMARY: The piriform muscle is a pelvitrochanteric muscle that gets its name due to its pear shape, whose origin are several fascicles located between the anterior foramina of the sacrum, corresponding to the second, third and fourth vertebrae. These fascicles are fused forming a flattened muscle, which is inserted into the greater trochanter of the femur. It has a well-known relationship with the sciatic nerve, which commonly emerges towards the gluteal region through the lower margin of this muscle, however over time, authors have described variations in the course of this nerve that could be associated with some compression pathology of the sciatic nerve. In a routine dissection of two formalized individuals, one female and one male, we found that the piriformis muscle originated through two heads, each with its own fascia that joined in a bicep-shaped common belly. Through a cylindrical tendon it is inserted into the medial part of the greater trochanter of the femur. The sciatic nerve was divided, the common fibular nerve emerged to the gluteal region through the heads, while in the tibial nerve divided through the inferior margin of the piriformis muscle. It is important to report on the anatomical variations to complement knowledge of these variations, which may explain certain physical and painful disorders such as the socalled piriformis muscle syndrome.

KEY WORDS: Piriformis muscle; Sciatic nerve; Variations.

Tabla I. Relación entre el músculo piriforme y nervio isquiático.

\begin{tabular}{llr}
\hline Tipos & Variaciones & $89,8 \%$ \\
\hline I & El nervio isquiático emerge debajo del músculo piriforme & $6,1 \%$ \\
II & Las divisiones del nervio isquiático pasan a través y debajo del músculo piriforme & $0,7 \%$ \\
& ("Se forma un músculo piriforme accesorio entre el nervio") & $0,7 \%$ \\
III & El nervio isquiático pasa a través y sobre el músculo piriforme & - \\
IV & El nervio isquiático pasa sobre el músculo piriforme & $0,7 \%$ \\
V & Las divisiones del nervio isquiático pasan sobre y debajo del músculo piriforme & $2 \%$ \\
VI & El nervio isquiático emerge completamente a través del músculo piriforme & \\
No clasificado & &
\end{tabular}




\section{REFERENCIAS BIBLIOGRÁFICAS}

Beaton, L. E. \& Anson, B. J. The relation of the sciatic nerve and of its subdivisions to the piriformis muscle. Anat. Rec., 70(1):1-5, 1937.

Boyajian-O`Neill, L. A.; McClain, R. L.; Coleman, M. K. \& Thomas, P. P. Diagnosis and management of piriformis syndrome: an osteopathic approach. J. Am. Osteopath. Assoc., 108(11):657-64, 2008.

Ilizaliturri, V. M.; Arriaga, R.; Villalobos, F. E. \& Suarez-Ahedo, C. Endoscopic release of the piriformis tendon and sciatic nerve exploration. J. Hip. Preserv. Surg., 5(3):301-6, 2018.

Jacomo, A. L.; Martinez, C. A. R.; Saleh, S. O.; Andrade, M. \& Akamatsu, F. E. Unusual relationship between the piriform muscle and sciatic, inferior gluteal and posterior femoral cutaneous nerves. Int. J. Morphol., 32(2):432-4, 2014.

Kirschner, J. S.; Foye, P. M. \& Cole, J. L. Piriformis syndrome, diagnosis and treatment. Muscle Nerve, 40(1):10-8, 2009.

Kraus, E.; Tenforde, A. S.; Beaulieu, C. F.; Ratliff, J. \& Fredericson, M. Piriformis syndrome with variant sciatic nerve anatomy: a case report. P.M. R., 8(2):176-9, 2016

Moore, K. L. \& Dalley, A. F. Anatomía con Orientación Clínica. $5^{\mathrm{a}}$ ed. Buenos Aires, Médica Panamericana, 2007.

Muñoz, S. Síndrome piriforme: Una controvertida neuropatía por atrapamiento. Rev. Med. Clin. Las Condes, 15(2):58-60, 2004.

Natsis, K.; Totlis, T.; Konstantinidis, G. A.; Paraskevas, G.; Piagkou, M. \& Koebke, J. Anatomical variations between the sciatic nerve and the piriformis muscle: a contribution to surgical anatomy in piriformis syndrome. Surg. Radiol. Anat., 36(3):273-80, 2014.

Polesello, G. C.; Queiroz, M. C.; Linhares, J. P. T.; Amaral, D. T. \& Ono, N. K. Anatomical variation of piriformis muscle as a cause of deep gluteal pain: diagnosis using MR neurography and treatment. Rev. Bras. Ortop., 48(1):114-7, 2013.

Ro, T. H. \& Edmonds, L. Diagnosis and management of piriformis syndrome: a rare anatomic variant analyzed by magnetic resonance imaging. J. Clin. Imaging Sci., 8:6, 2018.

Santos, C. M. T.; Pereira, C. U. \& Morais, A. A. Síndrome do piriforme: uma revisão da literatura. J. Bras. Neurocir., 20(1):46-52, 2009.

Testut, L. \& Latarjet, A. Tratado de Anatomía Humana. $9^{\mathrm{a}}$ ed. Barcelona, Salvat, 1972.

Vicente, E. J. D.; Viotto, M. J. S.; Barbosa, C. A. A. \& Vicente, P. C. Study on anatomical relationships and variations between the sciatic nerve and piriformis muscle. Rev. Bras. Fisioter., 11(3):227-32, 2007.
Dirección para correspondencia:

Gonzalo Tiznado Matzner

Universidad Austral de Chile

Campus Isla Teja S/N

Valdivia

CHILE

Email: gonzalo.tiznado@uach.cl

Recibido: $27-02-2019$

Aceptado:13-09-2019 